\title{
Motor Habits in Visuo-manual Tracking: Manifestation of an Unconscious Short-term Motor Memory?
}

\author{
ANDREAS HUFSCHMIDT, GÜNTHER DEUSCHL and CARL HERMANN \\ LÜCKING
}

Department of Neurology, University of Freiburg, Hansastrasse 9, D-7800 Freiburg i. Brsg., FRG.

Correspondence: $\operatorname{Dr}$ A. Hufschmidt, Neurologische Klinik, Hansastr. 9, D-7800 Freiburg, FRG.

\begin{abstract}
Normal subjects were tested in short, repetitive trials of a tracking task, with an identical shape of target movement being used throughout one session. Analysis of the net error curves (pursuit minus target movement) revealed that subjects regularly exhibit a remoteness effect: neighbouring trials were more similar than distant ones. The effect is demonstrated to be stronger in the absence of visual cues, and was found to be absent in a patient with complete loss of proprioception when he was performing without visual feedback as well.

The results are discussed in terms of a short term memory store contributing to unconscious movement habits in tracking. This may represent part of the motor learning process working together with conscious visuo-motor control mechanisms. Its function is probably related to the acquisition of automatic movements.
\end{abstract}

\section{Introduction}

Subjects who are tested on consecutive trials of a motor task tend to obtain similar scores in neighbouring trials. Thus, when inter-trial correlations of scores are arranged in a correlation matrix, they are highest near the diagonal and fall off with increasing distance. This "superdiagonal form" in practise matrices first attracted attention as early as 50 years ago (Viteles, 1933; Perl, 1934; Edgerton and Valentine, 1935; Greene, 1943). On the basis of factor-analytic studies, this effect was explained by the gradual emergence and disappearance of different skills in the course of motor learning, finally resulting in a stable simplification with the involvement of a small number of basic abilities (for discussion, see Jones, 1966). In the present study, an intraindividual remoteness effect is demonstrated in the way a subject performs a tracking task. Within a given session, similarity of tracking error curves is shown to be relatively high when trials performed in temporal vicinity are compared, becoming lower for trials laying further apart. We also demonstrate a recurrence of characteristic single errors as a parallel to the everyday phenomenon of error perseverance in motor sequences, which is familiar to musicians and secretaries: a typing error made, for instance, in the middle of a page has the fatal tendency to reoccur in exactly the same position when the page is retyped. We suggest that these motor habits develop under the influence of a memory store. The relation to other concepts of motor short-term memory is discussed. 


\title{
Subjects and Methods
}

\begin{abstract}
Subjects
Experiments were performed on healthy normal subjects (hospital staff; mean age 31.5 years, range $21 \ldots 56$ years), who volunteered to participate in the study. Subjects in experiment 1 had no previous experience in tracking.

Deafferentiation experiments were performed on a 37-year-old patient whose extremities were completely deafferented following an acute sensory neuronopathy (Sterman et al., 1980) 10 years ago. Sural nerve biopsy revealed an almost complete loss of myelinated nerve fibres with few remaining fibres of $5-10 \mu \mathrm{m}$ in diameter. Deafferation was demonstrated by repeated failure to elicit sensory nerve action potentials and somatosensory evoked potentials. Motor nerve conduction velocity was only moderately reduced. Tendon reflexes and joint position sense were completely lost. The patient experienced no severe disability as long as he was able to perform under visual control.
\end{abstract}

\section{Experimental set-up}

The subjects were seated before a TV screen on which the graphics for the tracking tasks were displayed by a microcomputer. An acoustic warning signal was presented $1 \mathrm{sec}$ before the onset of each trial. The task required pursuit tracking of a short vertical "target" bar moving horizontally across the screen, either sinusoidally or in a pseudo-random (Fig. 1a) fashion.

The position of a second "tracking" bar immediately below was controlled by the subject by means of a knob which they held between their thumb and index finger. Its axis was connected to a potentiometer. Transduction to the tracking bar was non-inverted and linear, a clockwise rotation of $40 \mathrm{deg}$ of the knob producing a horizontal displacement to the right of $8.5 \mathrm{~cm}$ of the tracking bar. The subject was instructed to keep the tracking bar aligned with the target bar. Movement amplitudes of the target bar were in the range of $8 \mathrm{~cm}$, subtending an angle of approximately $10 \mathrm{deg}$ of the subject's visual field. The target and tracking movement signals were digitized (8-bit) every $50 \mathrm{~ms}$ and stored by the microcomputer. The length of one trial was $12.8 \mathrm{~s}$ ( 256 points per channel). Trials were separated by a $2 \mathrm{~s}$ rest interval during which subjects were required to return the tracking bar to its starting position. One session involved 64 trials, all separately stored, with the same shape of target movement being used throughout the experiment.

\section{Experiments}

The following experiments were performed ( $n=$ number of subjects).

1 Pseudo-random pursuit tracking $(n=12)$. Pseudo-random signals were generated by superimposition of four sine waves $(0.2,0.27,0.35$, 


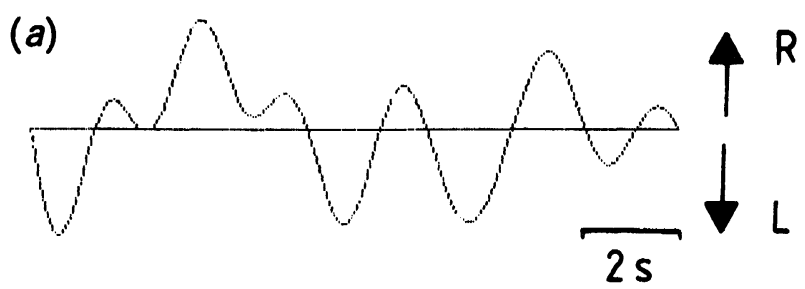

(b)

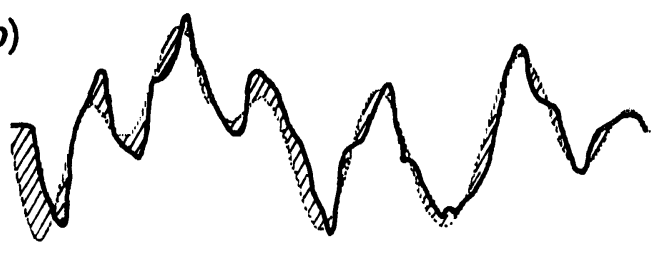

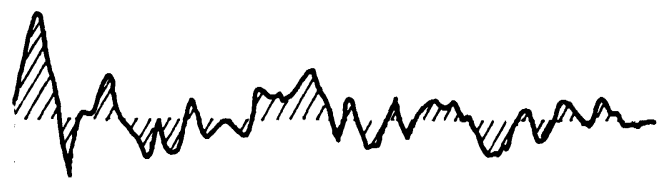

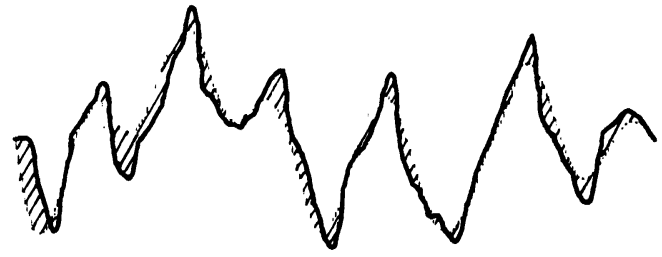

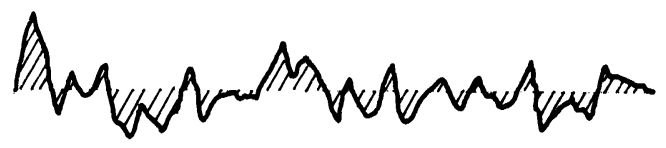

FIg. 1 (a) Target movement for random pursuit tracking. (b) Top traces: superposition of the target (thin line) and tracking curves (thick line); bottom traces: net "error" curves obtained by subtraction from the tracking of the target curve. Hatched area: tracking error.

$0.43 \mathrm{~Hz}$ ). Subjects were not informed that the pattern of target movements was the same in every trial, and, indeed, most of them did not notice it (see "Results"). This experiment was taken as a "low predictability" tracking task.

2 Sinusoidal pursuit tracking $(n=8)$, frequency $0 \cdot 18 \mathrm{~Hz}(2 \cdot 25$ cycles/ trial), with interrupted visual control by occlusion of the left half of the 
screen. Subjects were told in phases without visual feedback to imitate a sinusoid movement as well as possible. Thus, every trial of this task consisted of "high predictability" tracking segments (half-waves under visual guidance) serving as an instruction for "blind" segments where subjects had to perform an inverted half-wave under internal guidance.

Evaluation As a first step in off-line evaluation the target movement was subtracted from each of the tracking curves (Fig. 1b), thus rendering a "net" error curve. Quantitative evaluation was then performed as follows.

1. Tracking error The total error was computed as "modulus mean error" (average absolute error) by summing up the absolute difference between the target and tracking curve:

$$
\operatorname{Err}=\sum_{i=1}^{n}\left|x_{\mathrm{i}}\right|
$$

with $x_{\mathrm{i}} \ldots x_{\mathrm{n}}(n=256)$ being the single values of the error curve. To facilitate comparisons of different tracking tasks, error scores will be expressed as a percentage of the error in a "zero" trial in which the knob remained in its starting position.

2. Adjacent trial similarity The similarity between adjacent trials was measured by point-to-point linear regression of either the original tracking curves or the error curves. The results, of course, are different. The correlation coefficient $r$ serves as a measure of similarity.

3. Autocorrelation Autocorrelations were computed within single sessions to determine the similarity of tracking or error curves between the trials and its dependence on their proximity. Examples are displayed in Fig. 2. For the purpose of this study, a special type of autocorrelation was employed. Conventionally, a curve consisting of $n$ discrete values $\left(x_{1} \ldots x_{n}\right)$ is autocorrelated by the formula

$$
A(k)=\frac{1}{n-k} \sum_{i=1}^{n-k} x_{\mathrm{i}} \cdot x_{\mathrm{i}+\mathrm{k}}
$$

$k$ being the abscissa value of the current value $A(k)$ of the autocorrelation curve. The autocorrelation function is usually computed for values of $k=1$ $\ldots \frac{n}{2}$. Compared to the sum of the two values, the product $x_{\mathrm{i}} \cdot x_{\mathrm{i}+\mathrm{k}}$ in formula (2) is high when $x_{\mathrm{i}}$ and $x_{\mathrm{i}+\mathrm{k}}$ lie close together and low when they are very different or even have different signs. So, in the context of the autocorrelation function, this product measures the proximity of the two values relative to their sum. For the present study, autocorrelations were computed not for single points of a curve, but on the basis of single trials of an experimental session. Therefore, the product $x_{\mathrm{i}} \cdot x_{\mathrm{i}+\mathrm{k}}$ of two discrete values of a curve was 

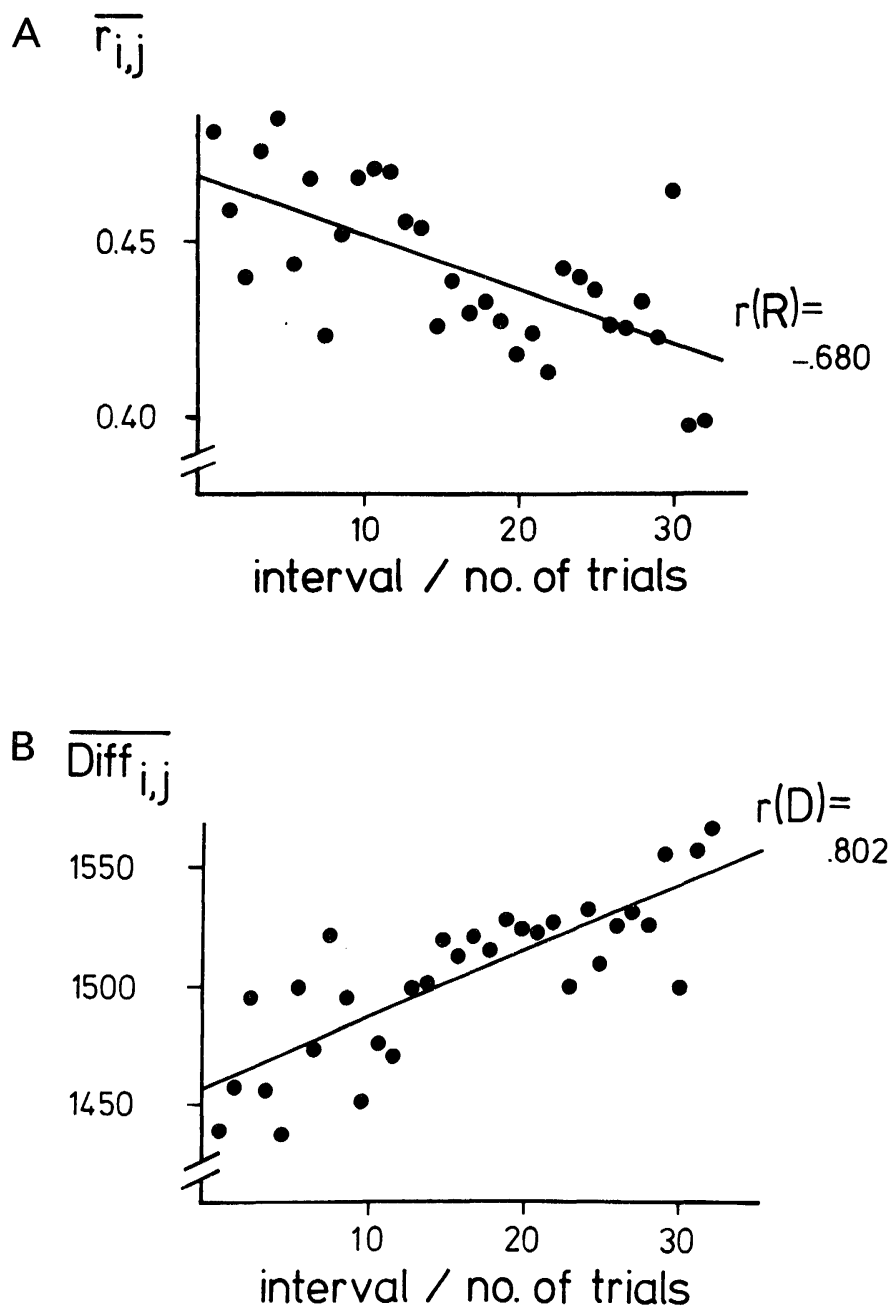

FIG. 2. Autocorrelation plots from single experiments (64 trials) of random pursuit tracking (experiment 1 , subject 1 in Table 1).

The error curves of trials within the experiment are compared either by point-to-point linear regression (A) or cumulated point-to-point difference (B). Abscissa: interval (in number of trials) between trials to be compared. Ordinates: mean correlation coefficient (A) and mean accumulated point-to-point difference $(B)$. The autocorrelation plots are approximated by linear regression, correlation coefficients in these experiments being significantly different from zero $(p<\cdot 001)$.

was replaced by a value indicating the "closeness" (in terms of similarity) of the error curves of trials $i$ (values $x_{\mathrm{il}} \ldots x_{\mathrm{in}}$ ) and $i$ (values $x_{\mathrm{j} 1} \ldots x_{\mathrm{jn}}$ ). Similarity was established by either point-to-point linear correlation of two error curves yielding a correlation coefficient $r_{\mathrm{i}, \mathrm{j}}$, or by summing up the point-to-point absolute difference 


$$
\operatorname{Diff}_{\mathrm{i}, \mathrm{j}}=\sum_{k=1}^{n}\left|x_{\mathrm{ik}}-x_{\mathrm{jk}}\right| .
$$

As a third autocorrelation parameter the product

$$
E r r_{\mathrm{i}, \mathrm{j}}=E r r_{\mathrm{i}} * E r r_{\mathrm{j}}
$$

of error scores of two trials was introduced, which in analogy to conventional autocorrelation indicates the "closeness" of error scores.

The first two parameters $\left(r_{\mathrm{i}, \mathrm{j}}\right.$ and $\left.D i f_{\mathrm{i}, \mathrm{j}}\right)$ measure the similarity of the tracking error curves, while $E r r_{i, j}$ serves to autocorrelate the total error scores.

The first point of the autocorrelation plot is obtained by comparing each trial to its successor. In an experiment involving 64 trials, 63 comparisons between adjacent trials are possible. The mean value resulting from these 63 comparisons is plotted as the leftmost point in the autocorrelogram (Fig. 2). The interval between the analyzed trials is then increased to 2, comparing Trial 1 to Trial 3, Trial 2 to Trial 4, and so forth. The number of possible comparisons is now only 62 . The resulting mean value yields the second point in the autocorrelation curve. This procedure is repeated with increasing step widths until the step width equals half the total number of trials (32) and the number of possible comparisons is down to 32. Thus, the formula for a single point in the autocorrelogram with an abscissa value (interval between trials to be compared) of $k$ is

$$
A_{r}(k)=\frac{1}{n-k} \sum_{i=1}^{n-k} r_{\mathrm{i}, \mathrm{i}+\mathrm{k}}
$$

( $n=$ number of trials) for the correlation coefficient serving as a measure of similarity, or accordingly

$$
A_{D i f f}(k)=\frac{1}{n-k} \sum_{i=1}^{n-k} D i f f_{\mathrm{i}, \mathrm{i}+\mathrm{k}}
$$

and

$$
A_{E r r}(k)=\frac{1}{n-k} \sum_{i=1}^{n-k} E r r_{\mathrm{i}, \mathrm{i}+\mathrm{k}}
$$

for the point-to-point difference and tracking error, respectively.

\section{Results}

Types of errors

Twelve inexperienced subjects were tested on a pseudo-random pursuit tracking task (experiment 1). Within-session comparison of the tracking 
error curves revealed that the subjects tended to make similar errors at similar points of the trials throughout the session ("local" errors). This is shown by the error curves of successive trials plotted in Fig. 3A and C. The errors were obviously related to the pitfalls (rapid segments, turns) of the target movement. After several sessions, this regularity dissolved into a pattern of prevailing random errors (Fig. $3 b, d)$. The total error was much lower at this stage. Quantitative data are presented in the next paragraph. In some sessions, errors at some of the pitfalls exhibited a tendency to wax and wane. This is best demonstrated in the row of initial error peaks of Fig. $3 a$, where two phases of large initial errors are separated by a temporary phase of improvement. Occasionally, unusually large local errors would be repeated either in the next trial (Fig. 4a) or some trials further on (Fig. 4b). The large errors at the beginning of trial 26 and 28 in Fig. $4 b$ are identical with the two peaks in Fig. 3a, marked by an arrow. The large "downward" errors towards the end of trials 25 and 28 are also unique within this set of curves, as is demonstrated by superimposition of all 32 trials (Fig. $4 b$, bottom).

Remoteness effect in error curve shape The observation of recurrent local errors was confirmed on a quantitative scale by autocorrelation of the error curves of experiment 1 . The resulting autocorrelation plot was approximated by a linear regression function (Fig. 2a,b). The slope of the curve was negative when point-to-point correlation coefficients $r_{\mathrm{i}, \mathrm{j}}$ were used to establish the similarity between the trials. It was positive when the point-to-point difference $D_{i f f}, \mathrm{j}$ was used, which in strict terms is a measure of dissimilarity.
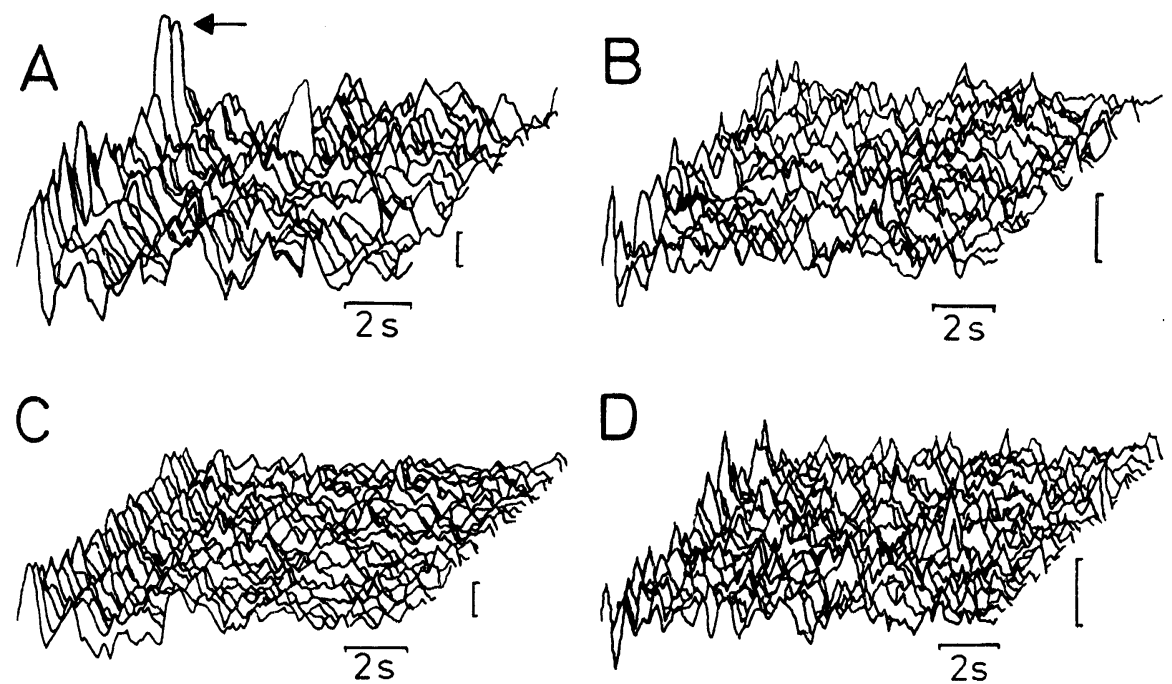

Fig. 3. Error curves from two subjects in their first $(A, C)$ and fourth (B,D) experimental sessions (experiment 1). The first 32 consecutive trials of each session are plotted. The first trials are the lowest. In the first sessions, a regular error pattern prevails, which with increasing skill is replaced by a random pattern. Arrow, see text. Note the different scale magnifying error curves in $\mathrm{B}$ and $\mathrm{D}$ by a factor of two. 
Trial

No

11

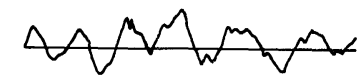

10
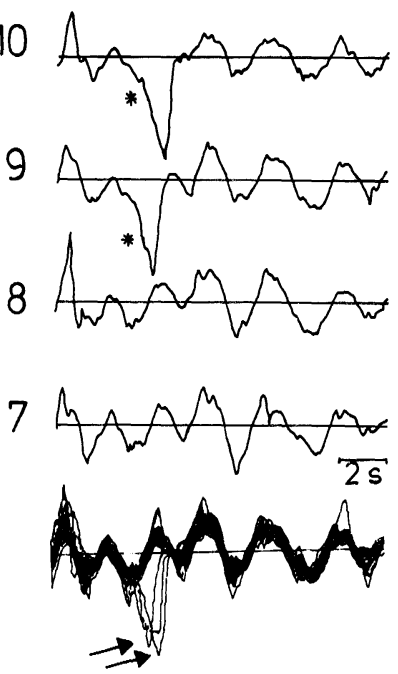

Trial

No B
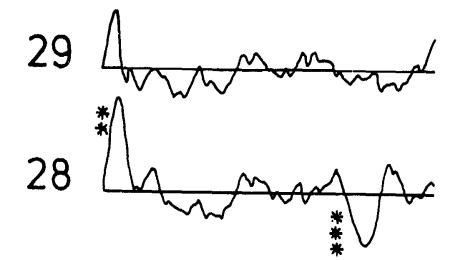

27

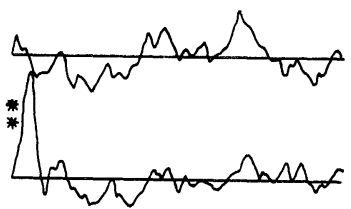

25

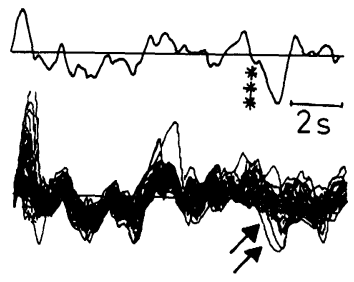

Fig. 4. Demonstration of single error curves with repetitive large errors in successive (A) or closely adjacent trials (B). The error marked * in trials 9 and $10(\mathrm{~A})$ is repeated only once (in trial 24) in the 32 successive trials superimposed on the bottom trace. In (B), error ** is repeated with one, error $* * *$ with two trials of normal shape in between. The error curves in (B) are taken from the same experiment shown in Fig. 3A, error ** being identical with the error marked by an arrow in Fig. 3A.

Quantitative data are shown in Table 1. These findings demonstrate a remoteness effect in the mode of execution of the tracking task. Similar errors are made in neighbouring trials and similarity decreases in trials further apart. As it turned out, the remoteness effect was not linked to a pseudo-random task, being present also with tracking of an entirely predictable pattern (experiment 2; see below). Subsequently, the absolute values of the correlation coefficients $r(\mathbf{R})$ and $r(\mathbf{D})$ resulting from linear regression of the two types of autocorrelation curves are taken as a measure of the strength of the remoteness effect.

Adjacent trial similarity of the error curves showed significant changes within the session in only four of the twelve subjects (no. 3, 8, 10, 12 in Table 1 ). In these cases, there was a decrease of similarity along the session, suggesting a decrease of regular errors (presumably at the pitfalls of the track) and a relative increase of random errors. This could represent a learning effect.

Long-term effects were studied by testing 10 of the original 12 subjects in five successive sessions. Intervals between the sessions ranged between 1 and 
TABLE 1. Tracking error and remoteness effect in random pursuit tracking (experiment $1, \mathrm{n}=12$ ).

\begin{tabular}{|c|c|c|c|c|c|c|c|}
\hline \multirow[b]{2}{*}{ Subject } & \multicolumn{2}{|c|}{$\begin{array}{l}\text { Relative tracking error } \\
(\%)\end{array}$} & \multicolumn{2}{|c|}{$\begin{array}{c}\text { Adjacent trial similarity } \\
\text { of original tracking } \\
\text { curves }\end{array}$} & \multicolumn{3}{|c|}{ Strength of remoteness effect } \\
\hline & $M$ & $r^{a}$ & $M$ & $r^{a}$ & $\mathrm{r}(\mathrm{R})$ & $\mathrm{r}(\mathrm{D})$ & $r(E)$ \\
\hline 1 & $28 \cdot 2$ & $-.59 * *$ & .96 & .02 & $-\cdot 68 * *$ & $\cdot 80 * *$ & $-.66 *$ \\
\hline 2 & $49 \cdot 8$ & $-\cdot 73 * *$ & $\cdot 86$ & $\cdot 24$ & $-\cdot 32$ & $62 * *$ & $-\cdot 86^{*}$ \\
\hline 3 & $28 \cdot 0$ & -.03 & .97 & $-\cdot 35 * *$ & $-\cdot 30$ & $\cdot 27$ & $-\cdot 39 *$ \\
\hline 4 & $36 \cdot 8$ & $-\cdot 21$ & .94 & $-\cdot 10$ & $-.57 * *$ & $\cdot 64 * *$ & $-\cdot 60^{*}$ \\
\hline 5 & $28 \cdot 1$ & $-.40 * *$ & $\cdot 96$ & -.03 & $-\cdot 76 * *$ & $\cdot 82 * *$ & $-.37 *$ \\
\hline 6 & $29 \cdot 0$ & $-\cdot 33 * *$ & $\cdot 96$ & $\cdot 09$ & $-\cdot 85^{* *}$ & $\cdot 67 * *$ & $-\cdot 87 *$ \\
\hline 7 & $28 \cdot 2$ & $\cdot 14$ & .95 & $-\cdot 11$ & $-\cdot 49 * *$ & $\cdot 56^{* *}$ & $-\cdot 66^{*}$ \\
\hline 8 & $53 \cdot 6$ & $\cdot 07$ & $\cdot 85$ & $-.26^{*}$ & $-.95 * *$ & $.92 * *$ & $-\cdot 29$ \\
\hline 9 & 28.6 & -.08 & .96 & $\cdot 18$ & $-.77 * *$ & $\cdot 75 * *$ & $-\cdot 16$ \\
\hline 10 & $43 \cdot 4$ & $-\cdot 14$ & .94 & $-.28^{*}$ & $-\cdot 75 * *$ & $\cdot 80 * *$ & $-.52 *$ \\
\hline 11 & $30 \cdot 1$ & $\cdot 16$ & .94 & $\cdot 11$ & $-\cdot 36^{*}$ & $\cdot 49 * *$ & $-.57^{*}$ \\
\hline 12 & $66 \cdot 4$ & $\cdot 01$ & $\cdot 65$ & $-.53 * *$ & $-.96 * *$ & $.97 * *$ & $-.92 *$ \\
\hline$M$ & $37 \cdot 5$ & & .91 & & -.65 & $\cdot 69$ & -.57 \\
\hline
\end{tabular}

${ }^{a}$ Rank correlation with trial number.

${ }^{6}$ Similarity measured (R) by point-to-point correlation of error curves, (D) by point-to-point difference. (E), remoteness effect expressed by autocorrelation of error scores.

$* p<\cdot 05,{ }^{* *} p<\cdot 01$.

10 days. Table 2 presents mean data from the first and fifth session, respectively. There was a significant reduction in tracking error and, in parallel, an increase in adjacent trial similarity of the original tracking curves, both obviously due to the subjects' growing ability to follow the target movements closely. The errors they made, however, were more irregular in the fifth session as shown by a decrease of adjacent trial similarity of net error curves. The effect is also illustrated by Fig. 3. The remoteness effect did not change significantly.

TABLE 2. Effect of five repetitive sessions on tracking performance, adjacent trial similarity and remoteness effect (mean values, $\mathrm{n}=10$ ).

\begin{tabular}{|c|c|c|c|c|}
\hline \multirow{3}{*}{ Relative tracking error $(\%)$} & \multicolumn{4}{|c|}{$\begin{array}{c}\text { Session No. } \\
\quad 1\end{array}$} \\
\hline & $M$ & $41 \cdot 0$ & * & $25 \cdot 6$ \\
\hline & $S D$ & $9 \cdot 9$ & & $7 \cdot 5$ \\
\hline Adjacent trial similarity & $M$ & $\cdot 90$ & * & .96 \\
\hline of original tracking curves ${ }^{a}$ & $S D$ & .06 & & .02 \\
\hline Adjacent trial similarity & $M$ & .57 & * & .36 \\
\hline of net error curves & ${ }^{a} S D$ & $\cdot 13$ & & $\cdot 08$ \\
\hline Strength of remoteness & $M$ & -.62 & & -.55 \\
\hline effect $(\mathbf{R})^{a}$ & $S D$ & $\cdot 27$ & & $\cdot 21$ \\
\hline Strength of remoteness & $M$ & $\cdot 68$ & & 62 \\
\hline effect $(\mathbf{D})^{b}$ & $S D$ & $\cdot 23$ & & $\cdot 17$ \\
\hline
\end{tabular}

a Similarity between trials measured by point-to-point correlation.

${ }^{b}$ Similarity measured by point-to-point difference.

$* p<\cdot 01$. 
Remarkably, most subjects, when questioned during or after the experiments, had not noticed that the same type of "random" movement was repeated in every trial. Some came up with suppositions that several types of movements were presented in turn, others believed that the movements were truly random except for their start segment. These subjective impressions were not, however, systematically investigated. The remoteness effect was not linked to an improvement of tracking performance and was also found in experiments in which total error did not change significantly or even increased slightly (subjects 7, 8, 9, 11, 12 in Table 1 ).

Remoteness effect in error score The tracking error score, too, tended to be similar in adjacent trials. When the autocorrelation was performed on the data of experiment 1 on the basis of the product of error scores $(r(E)$ in Table 1), a roughly linear decay of the autocorrelation curve was found in most experiments. This form indicates a remoteness effect of error score. In eight out of 12 subjects, the remoteness effect of error scores was weaker than the remoteness effect of error curve shape (Table 1). The remoteness effect of error scores was absent in subjects 8 and 9 who exhibited a highly significant remoteness effect of error curve shape. A few autocorrelation curves showed evidence of oscillations of tracking performance which were not visible in the original error score plots.

Dependence of remoteness effect on visual input The influence of visual cues was studied on eight normal subjects by testing them on a slow $(2.25$ cycles/trial) sinusoidal pursuit task with the left half of the monitor being obscured, restricting visual feedback to the right half (or "positive" half-waves) of the target curve (experiment 2; Fig. 5). Autocorrelation analysis was performed separately for each segment and showed significant autocorrelations. Numeric values are presented in Table 3.

When comparing the "blind" second half-wave to the visually guided third half-wave, correlation coefficients $r$ (D) of point-to-point differences were significantly higher $(p<\cdot 01)$ for the segment without visual feedback. This suggests that the remoteness effect is inhibited by visual control.

Dependence of remoteness effect on proprioceptive input The experiments were also performed on a deafferented patient (Table 4). His relative error score on pseudo-random pursuit (experiment 1 ) was exceptionally high (101\%). His tracking error curves exhibited a remoteness effect, but it was weaker than in normals (compare with Table 1) with $r(\mathbf{R})=-.30$ (below the $\cdot 05$ level of significance) and $r(\mathrm{D})=.56(p<\cdot 001)$. Mean adjacent trial similarity of original tracking curves was $r=\cdot 49$, of net tracking error curves $r=\cdot 38$, both values being below normal. The patient was also tested during the combined removal of proprioceptive and visual cues (slow sinusoidal pursuit with "blind" left half-waves; experiment 2). On separate analysis of the two "blind" segments of the track, no remoteness effect could be demonstrated (Table 4, columns 2 and 4).

In summary, the results from the experiments on a patient without 


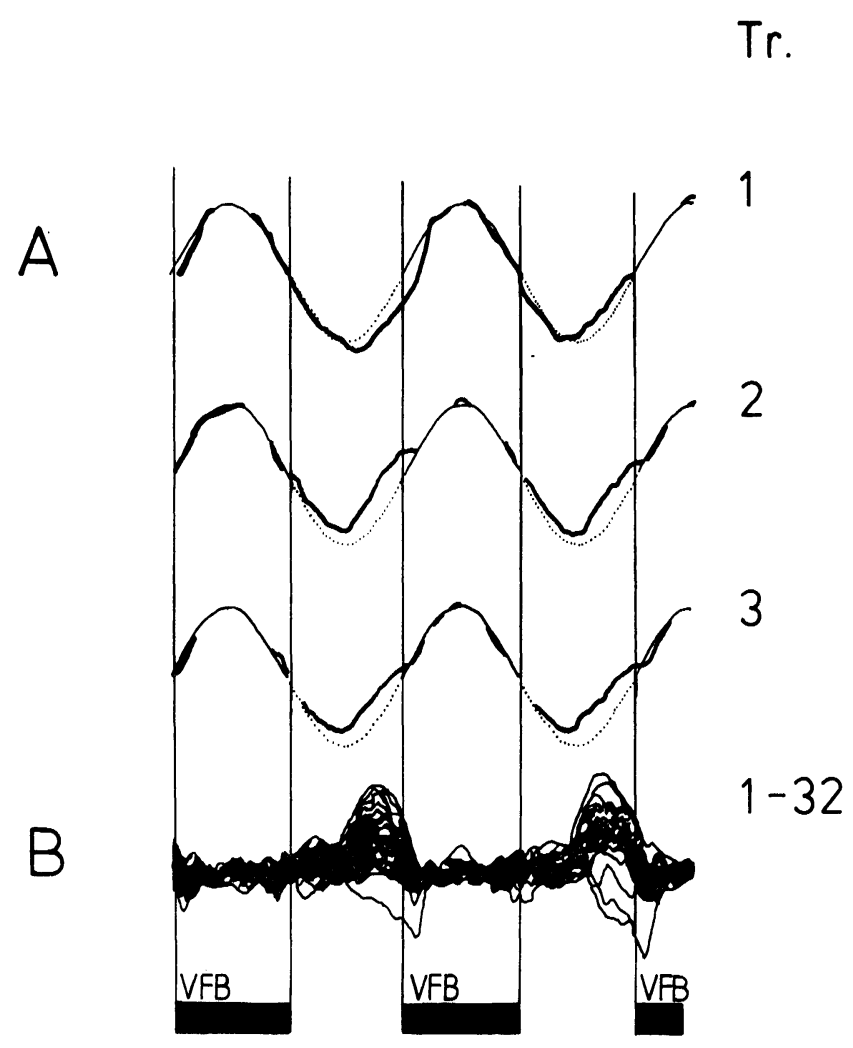

FIG. 5. (a) Single trials of a slow sinusoidal pursuit paradigm (experiment 2). Target movement: thin and dotted lines, pursuit: thick lines. Target movements to the right could be tracked by visual feedback (VFB). Target movements to the left were occluded from the subject's vision (dotted segments), leaving them to perform an arbitrary sinusoidal movement.

(b) Superposition of error curves (tracking minus target curves) of 32 trials, indicating superior performance under visual guidance.

proprioceptive sense appear to suggest that the remoteness effect is still present (but, if anything, weaker) in the absence of proprioceptive information. When both visual and proprioceptive cues were excluded, this patient exhibited no remoteness effect.

\section{Discussion}

The most prominent finding in the present study is an intraindividual remoteness effect between trials of a tracking task, which affects not only the total error score in the trials compared, but also the way movements are performed within the trials. Although we have analyzed the net error curves (target movement minus tracking response), the distance-dependent similarity is actually a similarity in movements, or, as we would prefer to say, a tendency for tracking behaviour to be partly determined by fluctuating 
TABLE 3. Effect of intermittent withdrawal of visual control on tracking error, adjacent trial similarity, and remoteness effect (experiment 2 , mean and $S D, \mathrm{n}=8$ )

\begin{tabular}{|c|c|c|c|c|c|}
\hline & & & $Q u$ & & \\
\hline & & 1 & 2 & 3 & 4 \\
\hline Visual control & & + & - & + & - \\
\hline Relative tracl & & & & & \\
\hline error $(\%)$ & $M$ & $15 \cdot 0$ & $42 \cdot 8$ & $20 \cdot 3$ & $41 \cdot 2$ \\
\hline & $S D$ & $4 \cdot 0$ & $20 \cdot 1$ & $7 \cdot 1$ & $18 \cdot 3$ \\
\hline Adjacent tria & & & & & \\
\hline $\begin{array}{l}\text { similarity of } \\
\text { error curves }^{a}\end{array}$ & $M$ & $\cdot 29$ & $\cdot 36$ & $\cdot 19$ & $\begin{array}{l}\cdot 39 \\
.21\end{array}$ \\
\hline $\begin{array}{l}\text { error curves } \\
\text { Strength of } \\
\text { remoteness ef }\end{array}$ & $S D$ & .05 & & & \\
\hline & $M$ & $-\cdot 31$ & -.42 & -.29 & $-\cdot 30$ \\
\hline & $S D$ & $-\cdot 28$ & $\cdot 30$ & $\cdot 19$ & $\cdot 36$ \\
\hline $\begin{array}{l}\text { Strength of } \\
\text { remoteness ef }\end{array}$ & & & & & \\
\hline & $M$ & $\cdot 26 *$ & $\cdot 48 * *$ & $\cdot 22$ & $\cdot 45$ \\
\hline & $S D$ & $\cdot 22$ & $\cdot 29$ & $\cdot 26$ & .42 \\
\hline
\end{tabular}

${ }^{a}$ Similarity between trials measured by point-to-point correlation.

${ }^{b}$ Similarity between trials measured by point-to-point difference.

$* p<\cdot 05, * * p<\cdot 01$.

TABLE 4. Effect of intermittent withdrawal of visual control on tracking in a deafferented patient (experiment 2).

\begin{tabular}{|c|c|c|c|c|}
\hline \multirow[b]{3}{*}{ Visual control } & \multicolumn{4}{|c|}{ Quarter } \\
\hline & 1 & 2 & 3 & 4 \\
\hline & + & - & + & - \\
\hline Relative tracking error $(\%)$ & $42 \cdot 2^{+}$ & $62 \cdot 4$ & $49 \cdot 1^{+}$ & $58 \cdot 8$ \\
\hline Adjacent trial similarity of original & & & & \\
\hline curves $^{a}$ & $62^{+}$ & 64 & $\cdot 62^{+}$ & $\cdot 63$ \\
\hline $\begin{array}{l}\text { Adjacent trial similarity of net error } \\
\text { curves }^{a}\end{array}$ & $\cdot 15$ & .20 & .09 & .20 \\
\hline Strength of remoteness effect $(\mathbf{R})^{a}$ & -.05 & .01 & $\cdot 04$ & .08 \\
\hline Strength of remoteness effect $(D)^{b}$ & $\cdot 45 * *$ & $-\cdot 19$ & $.37 *$ & $\cdot 15$ \\
\hline
\end{tabular}

${ }^{a}$ Similarity between trials measured by point-to-point correlation.

${ }^{b}$ Similarity between trials measured by point-to-point difference.

$+>M+3 S D$ of control group.

Significance of remoteness effect: $* p<\cdot 05, * * p<\cdot 01$.

habits. When the subjects are operating without visual feedback, the influence of these habits is greater. The discussion of these findings will centre around the following questions: (a) are there alternative explanations for the remoteness effect, (b) what is the relation of the suggested memory to the instance known as short term motor memory (STMM; Adams and Dijkstra, 1966), and (c) which sensory (or central motor) modalities contribute to the stored patterns? 
It is conceivable that fluctuations in attention may to some degree cause a remoteness effect: if tracking is performed equally well in adjacent trials, it may, for this reason only, be executed in a similar fashion. Remoteness effects as assessed by autocorrelation of the error scores of each trial along one session were, however, often weaker than those measured by autocorrelation of the shapes of error curves. Moreover, they were absent in two experiments with a significant autocorrelation of error curve shape. We conclude that varying levels of attention may theoretically contribute to the similarity in execution of trials in temporal vicinity, but this is not alone an adequate explanation.

Motor learning has been extensively studied by subjecting inter-trial correlations of scores to factor analysis (Perl, 1934; Edgerton and Valentine, 1935; Fleishman and Hempel, 1955) and other correlational methods (Jones, 1962). The resulting theory implies that motor skills are composed of a number of independent (basic) abilities, the relative importance of which changes continually in the course of learning. The skill finally resulting from practice is thought to be acquired by a reduction in the number of abilities employed. Considering the intraindividual remoteness effect in the experiments presented here, the application of this theory seems questionable. Firstly, the phenomenon of local errors recurring in close, though not always immediate, temporal vicinity is highly suggestive of an involuntary perseverance of previously executed movement patterns. If we were to try and explain this effect by changing abilities, it would be difficult to tell why a transient impairment of performance should recur at exactly the same segment within the trial. If, on the other hand, we postulate "local" abilities within the trials undergoing the same kind of modification, the current state of abilities for each segment of the trial would have to be stored somewhere. So, this interpretation actually involves a short-term memory store very similar to the one we are postulating. Secondly, our observation of a remoteness effect in some experiments without improvement in tracking accuracy would require at least two factors changing independently and, in these instances, with mutual compensation of their influence on tracking skill. Among the list of abilities suggested by Fleishman (1964), the one related closest to tracking is termed "rate control". It is difficult to imagine what other abilities should contribute to a task as straightforward as tracking or even tracking of an imagined movement without visual control as in experiment 2 .

The effects observed in this study lead us to suggest that the involvement of a short-term memory store is responsible for the remoteness effect and thus for the phenomenon of changing habits in tracking. If some information on previous trials is stored within the nervous system and does influence the execution of the present trial, we may first consider the relation of this memory store to conscious experience. Surprisingly, there seems to be none. Subjects did not realize that they were tracking the same movement in every trial, but behaved as if they did. Furthermore, they tended to repeat gross local errors like bad habits instead of avoiding them. Even if they were not able to verbalize local strategies, the assumption of any kind of conscious 
reproduction would be tenable only if subjects recognized that they were receiving the same stimulus in every trial. This dissociation is paralleled by findings in patients with complete loss of the ability to retain "declarative" information, who were still able to acquire motor skills (Corkin, 1968) and even more demanding ("procedural") problem-solving skills (Cohen et al., 1985). Previous research on short term motor memory (STMM) has largely centred on the ability to consciously reproduce active or passive movements under varying experimental conditions (Adams and Dijkstra, 1966; Posner, 1967; Marteniuk, 1973; Jones, 1974). The question arises: what is the relationship between the memory store suggested here and the STMM? As indicated above, the paradigms are fundamentally different, subjects being asked to retain and reproduce movements in the earlier experiments, while simply performing tracking without further instructions in the present condition. Furthermore, the inhibitory role of vision is particular to the remoteness effect and would, indeed, not make sense in voluntary movement reproduction.

Secondly, it might be asked whether the information stored is visual, proprioceptive, or motor, or any combination of these. Our occlusion experiments in normal subjects suggest that vision is not a necessary prerequisite and inhibits the remoteness effect rather than facilitating it. The influence of proprioceptive cues is more difficult to assess. Animal studies on deafferented neonate primates (Taub, 1977) showed that motor learning under these conditions is not severely impaired. Since isolated deafferentiation in man is a rare neurological condition, our data rest upon two experiments on a single patient and must therefore be interpreted with caution. In random pursuit tracking, the patient performed badly, with a remoteness effect weaker than normal. In segments of "predictable tracking without visual control" (or, more correctly, in segments requiring voluntary movement under the control of an internal plan; experiment 2), he exhibited no remoteness effect. There was a significant similarity between adjacent trials of the original error curves, suggesting that the patient did have an idea of how to move in segments without visual control. A patient with a similar decifit studied by Rothwell et al. (1982) also was quite able to perform pre-programmed finger movements. In the experiment on our patient, however, the similarity was not lower when regarding trials further apart. Obviously, the movement pattern was not modified, as in normals, by repetitive execution. The normal remoteness effect was preserved, however, in segments performed under visual control. In conclusion, this patient showed no evidence of a modifiable motor memory, in so far as it is manifested in the remoteness effect, when he was performing completely without feedback. This is evidence against a hypothetical contribution of efference copies to this memory store, which should be most efficient exactly in this situation. It is also in contrast to the findings of Lashley (1917), whose deafferented patient was indeed able to control and reproduce the rate and extent of leg movements, the difference being that these were movements under conscious voluntary control, whereas the remoteness effect reflects an unconscious influence of movement habits. We are careful, however, in 
applying conclusions from this experiment to physiological conditions, since our patient has certainly undergone extensive re-programming of his motor system to overcome his severe initial ataxia, in the course of which this particular mechanism may have proved useless and hence been suppressed. Adaptation may also explain the inverse influence on the remoteness effect of vision in this patient compared to normal subjects. All evidence taken together, the remoteness effect seems to be dependent on at least one source of feedback.

In conclusion, we suggest that the phenomenon of changing habits in tracking is related to the central storage of afferent and, perhaps, efferent signals, which in turn can substantially influence the reproduction of motor sequences. The fact that even single large errors are repeated suggests that this memory does not contain information on whether a movement is adequate or not. What could be the role of a mechanism for pure "motor perseverance" in the context of motor learning? Operating together with a conscious visuomotor control loop and/or the motor response schema proposed by Schmidt (1975), it would have to be programmed by repeated execution of adequate movements under the control of conscious feedback, shaping the stored patterns more and more towards an optimum. A gradual shift of motor control with increasing strength of a mechanism for pure movement reproduction may be the basis for the decrease of attentive demand observed with over-learning of a motor skill.

\section{Acknowledgement}

This work was supported by the Deutsche Forschungsgemeinschaft (SFB 325). The authors wish to thank Dr H. Heuer, Dr C. Wallesch and Dr S. Fellows for reviewing the manuscript as well as Miss D. Klingele and Miss P. van Wetten for technical assistance.

\section{References}

Adams, J. A. and Dijkstra, S. (1966). Short-term memory for motor responses. Journal of Experimental Psychology, 71, 314-318.

Cohen, N. J., Eichenbaum, H., Deacedo, B. S. and Corkin, S., (1985). Different memory systems underlying acquisition of procedural and declarative knowledge. Annals of the New York Academy of Sciences, 444, 54-71.

Corkin, S. (1968). Acquisition of motor skill after bilateral medial temporal-lobe excision. Neuropsychologia, 6, 255-266.

Edgerton, H. A. and Valentine, W. L. (1935). A factor analysis of learning data. Psychological Bulletin. 32, 719.

Fleishman, E. A. and Hempel, R. E. (1955). The relation between abilities and improvement with practice in a visual discrimination task. Fournal of Experimental Psychology, 49, 301312.

Fleishman, E. A. (1964). "The Structure and Measurement of Physical Fitness." PrenticeHall. Englewood Cliffs, NJ.

Greene, E. B. (1943). An analysis of random and systematic changes with practice. Psychometrica, 8, 37-53. 
Jones, B. (1974). Role of central monitoring of efference in short-term memory for movements. Fournal of Experimental Psychology, 102, 37-42.

Jones, M. B. (1962). Practice as a process of simplification. Psychological Review, 69, 274-294. Jones, M. B. (1966). Individual differences. In "Acquisition of Skill" (Ed. E. A. Bilodeau). Academic Press, New York, pp. 109-146.

Lashley, K. S. (1917). The accuracy of movement in the absence of excitation from the moving organ. American Journal of Physiology, 43, 169-194.

Marteniuk, R. G. (1973). Retention characteristics of motor short-term memory cues. Fournal of Motor Behavior, 5, 249-259.

Perl, R. E. (1934). An application of Thurstone's method for factor analysis to practice series. Journal of General Psychology, 11, 209-212.

Posner, M. I. (1967). Characteristics of visual and kinesthetic memory codes. Fournal of Experimental Psychology, 75, 103-107.

Rothwell, J. C., Traub, M. M., Day, D. L., Obeso, J. A., Thomas, P. K. and Marsden, C. D. (1982). Manual motor performance in a deafferented man. Brain, 105, 514-542.

Schmidt, R. A. (1975). A schema theory of discrete motor skill learning. Psychological Review, 82, 225-260.

Sterman, A. B., Schaumburg, H. H. and Asbury, A. K. (1980). The Acute Sensory Neuronopathy Syndrome: A Distinct Clinical Entity. Annals of Neurology, 7, 354-358.

Taub, E. (1977). Movement in nonhuman primates deprived of somatosensory feedback. In "Exercise and Sports Science Reviews". Vol. 4. (Ed. J. Keogh). Journal Publishing Affiliates, Santa Barbara.

Viteles, M. S. (1933). The influence of training on motor test performance. Fournal of Experimental Psychology, 16, 556-564.

Williams, H. L., Beaver, W. S., Spence, M. T. and Rundell, O. H. (1969). Digital and kinesthetic memory with interpolated information processing. Fournal of Experimental Psychology, 80, 530-536. 


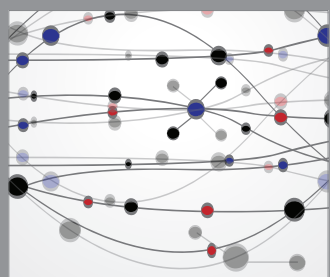

The Scientific World Journal
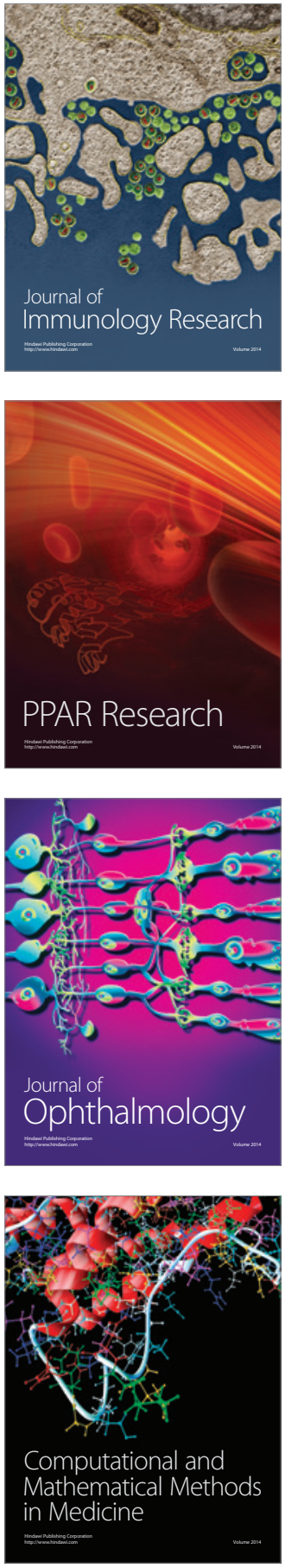

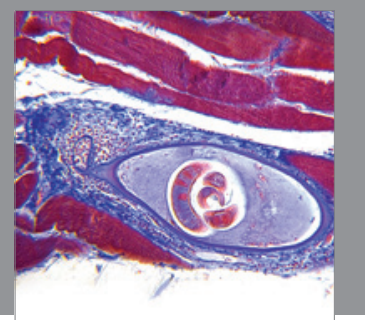

Gastroenterology

Research and Practice
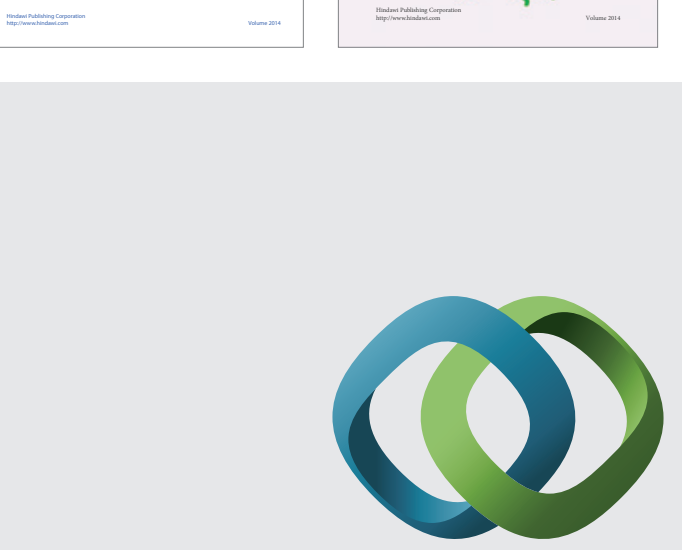

\section{Hindawi}

Submit your manuscripts at

http://www.hindawi.com
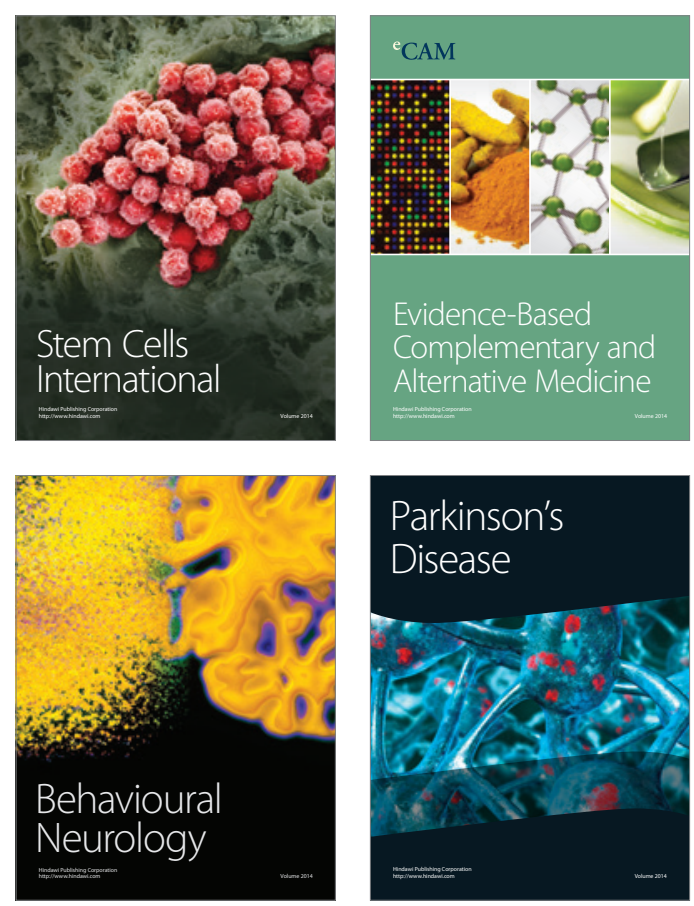

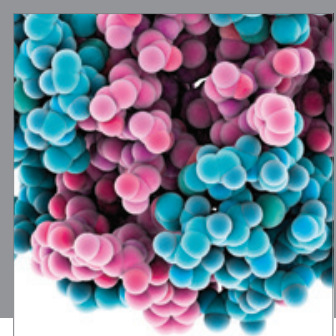

Journal of
Diabetes Research

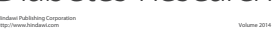

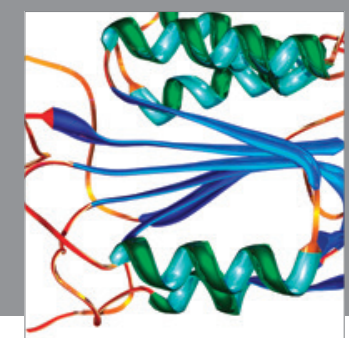

Disease Markers
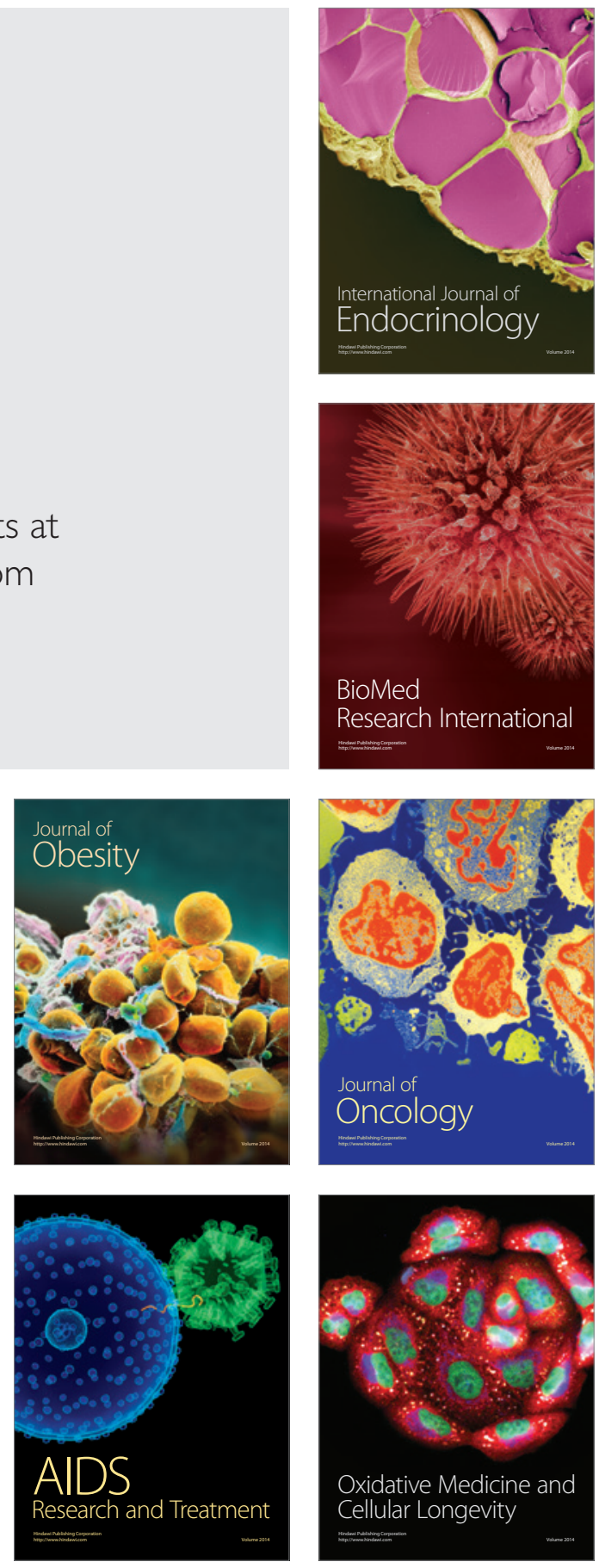